\title{
The Association between Cervical Collar and Intracranial Pressure Measured by the Optic Nerve Sheath Diameter in Trauma Patients Refered to the Emergency Department
}

\author{
Tamer Colak ${ }^{1, *}$, Kaan Celik ${ }^{1}$
}

\author{
${ }^{1}$ Bolu Abant Izzet Baysal University, \\ Medical Faculty Izzet Baysal Training and \\ Research Hospital, Emergency \\ Department, Turkey

\section{*Correspondence} \\ drtamercolak@gmail.com \\ (Tamer Colak)
}

\begin{abstract}
Objective: The aim of the present study was to determine the effect of the application or prolonged use of a Cervical Collar (CC) on intracranial pressure (ICP) through the changes in optic nerve sheath diameter (ONSD). Materials and methods: This prospective study was performed on 94 adult trauma patients who were referred to the emergency department between November, 15, 2017 and November, 15, 2018. Patients with any co-morbidities were excluded. The following factors were evaluated; age, gender, the mechanism of the trauma, the period between the trauma and admission, presence of any cranial and cervical trauma, symptoms and signs of head trauma, brain tomography (BT) findings, vital parameters, and ONSD values of the patients measured 3 times every 20 minutes. Results: The average age of the patients admitted to the emergency department due to trauma included 51 (54.3\%) males and was $42.0 \pm 16.1$ years. ONSD was $4.8 \pm 0.9 \mathrm{~mm}$ at admission, $5.0 \pm 0.9 \mathrm{~mm}$ at the 20th minute of the admission, and was $4.4 \pm 0.9 \mathrm{~mm}$ at the 20th minute after removal of the CC. The changes in ONSD diameter during the follow-up was statistically significant $(\mathrm{p}$ $<0.05)$. Conclusions: $\mathrm{CC}$ increases the ICP and ONSD regardless of the type of trauma types. Unnecessary CC applications should be avoided and the collar should be removed immediately after the presence of cervical trauma has been eliminated.
\end{abstract}

\section{Keywords}

Cervical collar, Intracranial pressure, Optic nerve sheath diameter, Trauma

\section{Introduction}

Head and neck injuries are commonly observed in trauma patients. The Advanced Trauma Life Support Manual recommends the use of a cervical collar (CC) in patients with suspected spinal injury or in whom spinal injury cannot be clearly evaluated [1,2].

Despite the belief that $\mathrm{CC}$ is not associated with any adverse effects, it has been reported that CC increases intracranial pressure (ICP) and it may negatively affect clinical outcomes $[3,4]$. The mechanism for the increase in ICP due to $\mathrm{CC}$ is thought to be increased pressure of $\mathrm{CC}$ on neck veins [4-6].

It has been reported that any condition which causes an increase in intracranial pressure in patients with head trauma may lead to secondary brain damage and negatively affect the patient's clinical condition [6].

Although invasive procedures for measurement of ICP improves outcomes, it is not possible to perform such procedures in an emergency department due to the concern for bleeding and local infection, and the necessity for special measurement devices [7]. For this reason, new diagnostic methods have been developed for identification of ICP. The optic nerve is a part of the central nervous system and is covered with dura and surrounded by subarachnoid cerebrospinal fluid (CSF) [7]. It has been reported that the optic nerve sheath diameter (ONSD) is increased in patients with an elevation in ICP $[8,9]$ and there is a significant 
TA B L E 1. Demographic and clinical features of patients.

\begin{tabular}{|c|c|c|c|}
\hline \multicolumn{2}{|l|}{ 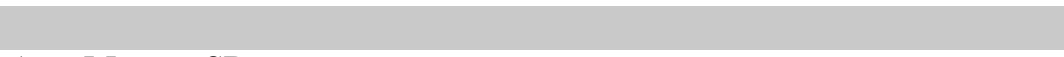 } & $n(\%)$ & Mean \pm SD/n (\%) \\
\hline Age, Mean \pm SD & & & $42.0 \pm 16.1$ \\
\hline \multirow{2}{*}{ Gender, $\mathbf{n}(\%)$} & Male & & $51(54.3 \%)$ \\
\hline & Female & & $43(45.7 \%)$ \\
\hline \multirow[t]{4}{*}{ Trauma mechanism, n(\%) } & In-vehicle traffic accident & & $44(46.8 \%)$ \\
\hline & Extravehicular traffic accident & & $14(14.9 \%)$ \\
\hline & Falls from height & & $26(27.7 \%)$ \\
\hline & Assault & & $10(10.6 \%)$ \\
\hline \multicolumn{3}{|c|}{ Duration of Access to the Hospital, Mean \pm SD } & $31.8 \pm 23.1$ \\
\hline \multirow[t]{5}{*}{ Symptom, n(\%) } & Altered state of consciousness & & $21(22.3 \%)$ \\
\hline & Nausea/vomiting & & $41(43.6 \%)$ \\
\hline & Headache & & $64(68.1 \%)$ \\
\hline & Hypotension & & $2(2.1 \%)$ \\
\hline & Bradycardia & & $4(4.3 \%)$ \\
\hline Head trauma & Present & & $73(77.7 \%)$ \\
\hline \multirow[t]{9}{*}{ Brain CT Pathology, n(\%) } & Present & & $30(31.9 \%)$ \\
\hline & Epidural hematoma & $2(6.7 \%)$ & \\
\hline & Subdural bleeding & $11(36.7 \%)$ & \\
\hline & Fracture & $16(53.3 \%)$ & \\
\hline & Subarachnoid hemorrhage & $5(16.7 \%)$ & \\
\hline & Parenchymal hematoma & $3(10 \%)$ & \\
\hline & contusion & $5(16.7 \%)$ & \\
\hline & Brain edema & $4(13.3 \%)$ & \\
\hline & Shift $5 \mathrm{~mm}$ & $5(16.7 \%)$ & \\
\hline \multicolumn{2}{|l|}{ Cervical trauma, $\mathbf{n}(\%)$} & & $50(53.2 \%)$ \\
\hline \multirow[t]{3}{*}{ GCS, $n(\%)$} & $3-8$ & & $11(11.7 \%)$ \\
\hline & $9-13$ & & $8(8.5 \%)$ \\
\hline & $14-15$ & & $75(79.8 \%)$ \\
\hline \multirow[t]{4}{*}{ Vital Sign, Mean \pm SD (0. Minutes) } & MAP & & $93.8 \pm 14.5$ \\
\hline & Pulse & & $77.8 \pm 14.7$ \\
\hline & Respiratory rate & & $17.2 \pm 3.7$ \\
\hline & Oxygen saturation & & $94.2 \pm 3.7$ \\
\hline \multirow[t]{4}{*}{ Vital Sign, Mean \pm SD (20. Minutes) } & MAP & & $93.5 \pm 14.2$ \\
\hline & Pulse & & $78.4 \pm 12.2$ \\
\hline & Respiratory rate & & $15.2 \pm 2.1$ \\
\hline & Oxygen saturation & & $97.1 \pm 2.1$ \\
\hline \multirow[t]{4}{*}{ Vital Sign, Mean \pm SD (40. Minutes) } & MAP & & $90.6 \pm 13.8$ \\
\hline & Pulse & & $82.3 \pm 11.8$ \\
\hline & Respiratory rate & & $11.9 \pm 1.4$ \\
\hline & Oxygen saturation & & $94.8 \pm 2.4$ \\
\hline \multirow[t]{3}{*}{ ONSD, Mean \pm SD } & 1.ONSD & $4.8 \pm 0.9$ & \\
\hline & 2.ONSD & $5.0 \pm 0.9$ & \\
\hline & 3.ONSD & $4.4 \pm 0.9$ & \\
\hline \multirow[t]{3}{*}{ Discharged Surgical intervention, n(\%) } & Discharged & & $58(61.7 \%)$ \\
\hline & Surgical intervention & & $8(8.5 \%)$ \\
\hline & Hospitalization & & $28(29.8 \%)$ \\
\hline
\end{tabular}

SD: standard deviation; CT: computerized tomography; ONSD: optic nerve sheath diameter; GCS: Glasgow coma scale; MAP: main arterial pressure.

relationship between ONSD increase and ICP [10, 11]. This expansion may be more clearly measured at the retrobulbar level [12].

The aim of the present study was to determine the effect of the application or prolonged use of $\mathrm{CC}$ on ICP and to demonstrate this impact through the increase in ONSD.

\section{Materials and methods}

This study was performed in 94 trauma patients over the age of 18 years who were referred to the Emergency Department of Izzet Baysal Training and Research Hospital affiliated with the Abant Izzet Baysal University between 
TA B L E 2. The relationship between demographic characteristics of the patients and ONSD values/alterations.

\begin{tabular}{|c|c|c|c|c|c|c|}
\hline & & n (\%) & $\begin{array}{c}\text { 1.ONSD } \\
\text { Mean } \pm \text { SD }\end{array}$ & $\begin{array}{c}\text { 2.ONSD } \\
\text { Mean } \pm \text { SD }\end{array}$ & $\begin{array}{c}\text { 3.ONSD } \\
\text { Mean } \pm \text { SD }\end{array}$ & $\mathbf{p}$ \\
\hline All patients & & & $4.8 \pm 0.9^{a c}$ & $5.0 \pm 0.9^{a b}$ & $4.4 \pm 0.9^{b c}$ & $<0.001$ \\
\hline \multirow[t]{2}{*}{ Gender } & Male & $51(54.3 \%)$ & $4.9 \pm 1.0^{a c}$ & $5.1 \pm 1.0^{a b}$ & $4.5 \pm 1.0^{b c}$ & $<0.001$ \\
\hline & Female & $43(45.7 \%)$ & $4.7 \pm 0.9^{a c}$ & $4.9 \pm 0.8^{a b}$ & $4.3 \pm 0.8^{b c}$ & \\
\hline \multirow[t]{4}{*}{ Mechanism of trauma } & In-vehicle traffic accident & $44(46.8 \%)$ & $4.9 \pm 0.9^{a c}$ & $5.1 \pm 0.9^{a b}$ & $4.4 \pm 0.9^{b c}$ & $<0.001$ \\
\hline & Extravehicular traffic accident & $14(14.9 \%)$ & $4.6 \pm 0.9^{a c}$ & $4.9 \pm 0.9^{a b}$ & $4.3 \pm 0.9^{b c}$ & $<0.001$ \\
\hline & Falls from height & $26(27.7 \%)$ & $5.0 \pm 1.0^{a c}$ & $5.2 \pm 1.0^{a b}$ & $4.6 \pm 1.1^{b c}$ & $<0.001$ \\
\hline & Assault & $10(10.6 \%)$ & $4.3 \pm 0.6^{a c}$ & $4.4 \pm 0.7^{a b}$ & $3.8 \pm 0.6^{b c}$ & $<0.001$ \\
\hline \multirow[t]{2}{*}{ Head trauma } & Present & $73(77.7 \%)$ & $4.9 \pm 1.0^{a c}$ & $5.1 \pm 1.0^{a b}$ & $4.5 \pm 0.9^{b c}$ & $<0.001$ \\
\hline & Absent & $21(22.3 \%)$ & $4.4 \pm 0.6^{a c}$ & $4.6 \pm 0.6^{a b}$ & $3.9 \pm 0.5^{b c}$ & $<0.001$ \\
\hline \multirow[t]{2}{*}{ Pathology detected by CT } & Present & $30(31.9 \%)$ & $5.8 \pm 0.8^{a c}$ & $5.9 \pm 0.7^{a b}$ & $5.4 \pm 0.7^{b c}$ & $<0.001$ \\
\hline & Absent & $48(51.1 \%)$ & $4.4 \pm 0.6^{a c}$ & $4.6 \pm 0.6^{a b}$ & $3.8 \pm 0.5^{b c}$ & $<0.001$ \\
\hline \multirow[t]{2}{*}{ Altered state of consciousness } & Present & $21(22.3 \%)$ & $5.8 \pm 0.8^{a c}$ & $6.0 \pm 0.7^{a b}$ & $5.5 \pm 0.8^{b c}$ & $<0.001$ \\
\hline & Absent & $73(77.7 \%)$ & $4.5 \pm 0.7^{a c}$ & $4.7 \pm 0.7^{a b}$ & $4.1 \pm 0.7^{b c}$ & $<0.001$ \\
\hline \multirow[t]{2}{*}{ Nausea/vomiting } & Present & $41(43.6 \%)$ & $5.3 \pm 1.1^{a c}$ & $5.5 \pm 1.1^{a b}$ & $4.9 \pm 1.0^{b c}$ & $<0.001$ \\
\hline & Absent & $53(56.4 \%)$ & $4.5 \pm 0.6^{a c}$ & $4.7 \pm 0.6^{a b}$ & $3.9 \pm 0.6^{b c}$ & $<0.001$ \\
\hline \multirow[t]{2}{*}{ Headache } & Present & $64(68.1 \%)$ & $5.1 \pm 0.9^{a c}$ & $5.3 \pm 0.9^{a b}$ & $4.7 \pm 1.0^{b c}$ & $<0.001$ \\
\hline & Absent & $30(31.9 \%)$ & $4.2 \pm 0.5^{a c}$ & $4.4 \pm 0.6^{a b}$ & $3.8 \pm 0.5^{b c}$ & $<0.001$ \\
\hline \multirow[t]{2}{*}{ Hypotension } & Present & $2(2.1 \%)$ & $6.3 \pm 0.4$ & $6.6 \pm 0.7$ & $6.1 \pm 0.7$ & 0.326 \\
\hline & Absent & $92(97.9 \%)$ & $4.8 \pm 0.9^{a c}$ & $5.0 \pm 0.9^{a b}$ & $4.5 \pm 0.9^{b c}$ & $<0.001$ \\
\hline \multirow[t]{2}{*}{ Bradycardia } & Present & $4(4.3 \%)$ & $6.6 \pm 0.5$ & $6.6 \pm 0.3$ & $6.3 \pm 0.6$ & 0.055 \\
\hline & Absent & $90(95.7 \%)$ & $4.7 \pm 0.9^{a c}$ & $4.9 \pm 0.9^{a b}$ & $4.3 \pm 0.8^{b c}$ & $<0.001$ \\
\hline \multirow[t]{3}{*}{ Result } & Discharged & $58(61.7 \%)$ & $4.3 \pm 0.6^{a c}$ & $4.5 \pm 0.6^{a b}$ & $3.9 \pm 0.5^{b c}$ & $<0.001$ \\
\hline & Surgical intervention & $8(8.5 \%)$ & $5.9 \pm 0.9^{a c}$ & $6.1 \pm 0.7^{a b}$ & $5.6 \pm 0.9^{b c}$ & $<0.001$ \\
\hline & Hospitalization & $28(29.8 \%)$ & $5.5 \pm 0.8^{a c}$ & $5.7 \pm 0.8^{a b}$ & $5.2 \pm 0.8^{b c}$ & $<0.001$ \\
\hline
\end{tabular}

$a: p<0.05$ for ONSD 1 and ONSD 2; $b: p<0.05$ for ONSD 2 and ONSD 3; $c: p<0.05$ for ONSD 1 and ONSD 3. ONSD: optic nerve sheath diameter, CT: computerized tomography.

November, 15, 2017 and November, 15, 2018. The study was initiated after approval of the local ethics committee of Izzet Baysal Training and Research Hospital. All participants were adults and provided consent for the study.

The exclusion criteria included the following; not willing to give consent or to share medical information due to any reason, patients less than 18 years of age, any previous cerebrovascular event (CVE) or trans-ischemic attack (TIA), hypertensive encephalopathy, vascular dementia, liver insufficiency and chronic kidney failure, pregnancy or breastfeeding, optic neuritis, arachnoid cyst in the optic nerve and a mass lesion in the orbit/cavernous sinus. The patients in whom a fracture and/or spinal injury were confirmed by direct cervical X-ray or cervical CT were excluded due to the inability to perform all serial ONSD measurements.

The following factors were evaluated; age, gender, the mechanism of the trauma, the period between the trauma and admission, presence of any cranial and cervical trauma, symptoms and signs of head trauma, brain tomography
(BT) findings, vital parameters (ONSD measurements simultaneously at 20th and 40th minutes), and ONSD values measured 3 times every 20 minutes.

The first ONSD measurement was performed at the time of admission. The second measurement was performed in those patients with CC in whom cervical fracture and/or spinal injury were not suspected after 20 minutes following admission. The $\mathrm{CC}$ was removed at the end of the 20th minute and the third measurement was performed after the patients were followed up for an additional 20 minutes. When any pathology such as a ligament injury was suspected in those patients with persistent cervical motion tenderness, a Philadelphia- collar was placed after the measurement.

The ONSD measurement was performed by a welltrained emergency medical specialist using a $7.5 \mathrm{mHz}$ linear probe following application of a thin layer of gel over both eyes in a supine position. ONSD was measured $3 \mathrm{~mm}$ posterior to the globe at sagittal and transverse positions. The average of ONSD was determined by calculation of 
the average of the transverse and sagittal measurements of the right and left ONSD.

The data were analyzed by SPSS Windows 22.0 version. The distribution of the variables was controlled through Kolmogorov Smirnov Test. The descriptive statistical analyses of the data were expressed as an average, standard deviation, and frequency. The student-t-test was used to compare the numerical parametric data whereas non-parametric data were evaluated by the Mann Whitney U test. Pearson's correlation analysis was performed for comparison of quantitative data; Pearson's chi-square test and Fisher's exact chi-square test were used for analysis of qualitative data. Repeated clinical measurements were performed by the Repeated Measures Analysis of Variance. The significance of Group x Time interaction was evaluated through Greenhouse-Geisser test statistics. When the results of Greenhouse-Geisser test statistics were significant, a paired sample test was used for determination of the parameters leading to significance. The $p$ values below $0.05(p<0.05)$ were accepted as statistically significant unless otherwise stated.

\section{Results}

The age average of the patients who were admitted to the emergency department due to trauma included 51 males $(54.3 \%+/-9)$ and was $42.0 \pm 16.1$ years. The most common cause for admission was motor vehicle traffic accidents (46.8\%). Demographic data and clinical findings of the patients are presented in Table 1 . ONSD was $4.8 \pm$ $0.9 \mathrm{~mm}$ at admission, $5.0 \pm 0.9 \mathrm{~mm} 20$ minutes following the admission, and $4.4 \pm 0.9 \mathrm{~mm}$ after 20 minutes following removal of the $\mathrm{CC}$ (Table 1 ).

The alteration in ONSD diameter during the follow-up was statistically significant $(p<0.05)$. ONSD was significantly higher after 20 minutes following the admission than the diameter detected at admission. ONSD decreased significantly after removal of the $\mathrm{CC}$; however, the value obtained after removal of the $\mathrm{CC}$ was significantly higher than the values recorded at the time of admission $(p<0.05)$ (Table 2).

The alterations in ONSD values were statistically significant in both genders; in all types of trauma; in the presence and absence of head trauma, cerebral pathology detected by $\mathrm{CT}$, altered state of consciousness, nausea/vomiting, headache; and in the absence of hypotension and bradycardia $(\mathrm{p}<0.05)$. The alterations in ONSD in patients with hypotension and bradycardia were not significant $(p$ $>0.05$ ) (Table 2)

There was a positive correlation between the ONSD values detected at admission and subsequent changes in mean arterial pressure (MAP) and respiratory rate following hospital admission. However, ONSD values were negatively correlated with Glasgow coma score (GCS) and oxygen saturation $(p<0.05)$. No correlation was observed between the ONSD pulse rate and temperature $(\mathrm{p}>0.05)$ (Table 3).

ONSD was found to be higher in the patients with head trauma, cerebral pathology detected by CT, altered state of consciousness, nausea/vomiting, headache, hypotension and bradycardia $(\mathrm{p}<0.05)$. No correlation was detected between the ONSD values and the presence of cervical pathology at all three measurement periods $(p>0.05)$ (Table 4).

\section{Discussion}

Increased ICP results in tissue damage by impairing cerebral perfusion. Therefore, the early identification of ICP is essential to prevent further brain damage [13-15]. ONSD may be easily evaluated through ultrasonography, and has a strong correlation with ICP [15-17]. Ohle et al. reported in their meta-analysis that ONSD has higher negative and positive predictive value rates to demonstrate ICP, with a sensitivity and specifity rates of $95.6 \%$ and $92.3 \%$, respectively [16].

Stone et al. [18] and Karason et al. [19] found that CC causes fluid accumulation in the intracranial area by direct compression of the jugular veins which eventually increases in ICP. Raphael et al. reported that Stiffneck-type CCs applied during spinal anaesthesia cause alterations in the intracranial pressure and impairs the drainage of the cerebral sinus through the pressure on the jugular vein which acts as a valve, causing an increase in the subarachnoid CSF pressure [20]. Kroeker et al. reported the CC increases the ICP, however the venous return becomes normal and ICP decreases within five minutes after removal of the CC [21]. In our study, an increase in ONSD thickness was detected in the patients using a $\mathrm{CC}$; and the rate of the thickness decreased after 20 minutes following removal of the CC; however, the ONSD thickness did not return to the initial value even after 20 minutes. This was not associated with gender, trauma types, head/brain trauma, developing symptoms (altered state of consciousness, headache, nausea/vomiting) and emergency outcomes. ONSD alterations were not significant in patients with hypotension and bradycardia. We believe that the $\mathrm{CC}$ causes blood collection in the intracranial area by compressing the jugular vein in the neck and results in increased intracranial damage; independent of the mechanism of trauma and the patients presenting symptoms. The absence of changes in the patients with bradycardia and hypotension may be related to the limited number of cases. Since minor circulation disorders inside the cranium may cause severe circulation disorders, especially for the patients with intracranial injury and without cervical injury, using a $\mathrm{CC}$ in these patients would unnecessarily contribute to the increase in ICP which is already elevated. We therefore believe that the $\mathrm{CC}$ should be removed as soon as possible in these patients. Alternative stabilization methods which do not impair venous blood return may be preferred in these patients with a cervical injury.

Mobbs et al. found that the heart rate and blood pressure were not affected by CC application [6]. Davies et al. reported that mean arterial pressure did not change before and after the $\mathrm{CC}$ application and there was no correlation 
TA B L E 3. The association of ONSD with duration of access to hospital and vital parameters.

\begin{tabular}{lcccccc} 
& \multicolumn{2}{c}{ 1.ONSD } & \multicolumn{2}{c}{$\mathbf{2 . O N S D}$} & \multicolumn{2}{c}{ 3.ONSD } \\
& $\mathbf{r}$ & $\mathbf{p}$ & $\mathbf{r}$ & $\mathbf{p}$ & $\mathbf{~ r}$ & $\mathbf{p}$ \\
Duration of Access to the Hospital. & 0.215 & 0.038 & 0.243 & 0.019 & 0.211 & 0.041 \\
GCS & -0.514 & 0 & -0.548 & 0 & -0.581 & 0 \\
MAP & 0.354 & 0 & 0.349 & 0.001 & 0.296 & 0.004 \\
Pulse & 0.069 & 0.506 & 0.032 & 0.756 & 0.072 & 0.491 \\
Respiratory rate & 0.422 & 0 & 0.217 & 0.036 & 0.273 & 0.008 \\
Oxygen saturation & -0.413 & 0 & -0.251 & 0.015 & -0.208 & 0.045 \\
\hline
\end{tabular}

ONSD: optic nerve sheath diameter; GCS: Glasgow coma scale; MAP: main arterial pressure.

T A B L E 4. The association of ONSD with symptoms and findings.

\begin{tabular}{|c|c|c|c|c|c|}
\hline & & $\begin{array}{l}\text { Number of cases } \\
\text { Present/ Absent }\end{array}$ & Present Mean \pm SD & Absent Mean \pm SD & $\mathbf{p}$ \\
\hline \multirow[t]{8}{*}{ ONSD 1} & Head injury & $73 / 21$ & $4.9 \pm 1.0$ & $4.4 \pm 0.6$ & 0.009 \\
\hline & Cerebral Pathology detected by CT & $30 / 48$ & $5.8 \pm 0.8$ & $4.4 \pm 0.6$ & $<0.001$ \\
\hline & Altered state of consciousness & $21 / 73$ & $5.8 \pm 0.8$ & $4.5 \pm 0.7$ & $<0.001$ \\
\hline & Nausea/vomiting & $41 / 93$ & $5.3 \pm 1.1$ & $4.5 \pm 0.5$ & $<0.001$ \\
\hline & Headache & $64 / 30$ & $5.1 \pm 0.9$ & $4.2 \pm 0.5$ & $<0.001$ \\
\hline & Hypotension & $2 / 92$ & $6.3 \pm 0.4$ & $4.8 \pm 0.9$ & 0.024 \\
\hline & Bradycardia & $4 / 94$ & $6.6 \pm 0.5$ & $4.7 \pm 0.9$ & $<0.001$ \\
\hline & Result & $36 / 58$ & $5.6 \pm 0.8$ & $4.3 \pm 0.6$ & $<0.001$ \\
\hline \multirow[t]{8}{*}{ ONSD 2} & Head injury & $73 / 21$ & $4.6 \pm 1.0$ & $5.1 \pm 01.0$ & 0.014 \\
\hline & Cerebral Pathology detected by CT & $30 / 48$ & $6.0 \pm 0.7$ & $4.6 \pm 0.6$ & $<0.001$ \\
\hline & Altered state of consciousness & $21 / 73$ & $6.0 \pm 0.7$ & $4.7 \pm 0.7$ & $<0.001$ \\
\hline & Nausea/vomiting & $41 / 93$ & $5.5 \pm 1.1$ & $4.7 \pm 0.6$ & $<0.001$ \\
\hline & Headache & $64 / 30$ & $5.3 \pm 0.9$ & $4.4 \pm 0.6$ & $<0.001$ \\
\hline & Hypotension & $2 / 92$ & $6.6 \pm 0.7$ & $5.0 \pm 0.9$ & 0.011 \\
\hline & Bradycardia & $4 / 94$ & $6.6 \pm 0.5$ & $4.9 \pm 0.8$ & $<0.001$ \\
\hline & Result & $5.8 \pm 0.8$ & $4.5 \pm 0.6$ & & $<0.001$ \\
\hline \multirow[t]{8}{*}{ ONSD 3} & Head injury & $73 / 21$ & $4.5 \pm 1.0$ & $3.9 \pm 0.5$ & 0.008 \\
\hline & Cerebral Pathology detected by CT & $30 / 48$ & $5.4 \pm 0.7$ & $3.9 \pm 0.5$ & $<0.001$ \\
\hline & Altered state of consciousness & $21 / 73$ & $5.5 \pm 0.7$ & $4.1 \pm 0.7$ & $<0.001$ \\
\hline & Nausea/vomiting & $41 / 93$ & $4.9 \pm 1$ & $4.0 \pm 0.6$ & $<0.001$ \\
\hline & Headache & $64 / 30$ & $4.7 \pm 0.9$ & $3.8 \pm 0.5$ & $<0.001$ \\
\hline & Hypotension & $2 / 92$ & $6.1 \pm 0.7$ & $4.3 \pm 0.9$ & 0.007 \\
\hline & Bradycardia & $4 / 94$ & $6.3 \pm 0.6$ & $4.3 \pm 0.8$ & $<0.001$ \\
\hline & The result (hospitalization) & $36 / 58$ & $5.2 \pm 0.8$ & $3.9 \pm 0.5$ & $<0.001$ \\
\hline
\end{tabular}

ONSD: optic nerve sheath diameter; CT: computerized tomography.

between arterial blood pressure and ICP [4]. It was reported that the increase in ICP may be caused by an increase of blood, brain and CSF pressures and such an increase may result in respiratory difficulties, and an elevation in the blood pressure. [22]. In the present study, it was detected that ONSD was positively correlated with MAP and respiratory rate, and negatively correlated with GCS and oxygen saturation. Anxiety caused by the increases in pain and blood pressure as a result of the increased ICP may lead to the changes in respiratory rate. This condition may also explain the correlation imbalance between saturation and ONSD. It should be noted that vital signs may be altered in order to preserve cerebral perfusion disrupted by the severity of the trauma, and that these altered vital signs (respiration count and oxygen saturation) may decrease the oxygen saturation of the blood and thereby the ONSD 
in traumatic brain injuries. Our data are consistent with previous studies showing than an increase in the severity of the intracranial injury results in an increase in ICP and decrease in GCS. The increased ICP may reduce the GCS, and these patients may be intubated due to lower GSC which impairs the interaction between vital signs and ICP.

Previous studies have reported that head trauma increases the ICP and that this increase is exacerbated by a CC [3$6,23]$. The increase in ICP is characterized by headache, nausea, vomiting, dilatation of the pupils, impaired consciousness, papillary oedema, and decerebrate/decorticate posturing [22]. In our study, the ONSD rates were significantly higher in the patients with an intracranial lesion and head trauma or those who were admitted due to the symptoms associated with these lesions. This condition may be associated with an increase in the ICP caused by the intracranial lesion itself. We believe that the impaired intracranial circulation caused by trauma is exacerbated in these patients and increases ONSD.

Wooster et al. reported that ONSD started to increase within 5 minutes after $\mathrm{CC}$ application and such an increase continued until the 20th minute of application [15]. In this study, a significantly positive correlation was found between the duration of hospital admission and ONSD. This may be related to the CC-dependent increase over time. Our hypothesis is supported by the difference between the ONSD value at admission to the ED and the value at the 20th minute. This may also be related to the decrease in venous return in the head caused by the intracranial injuries in some of these patients.

The most significant limitation of the present study was the inability to measure the ONSD levels of the patients within the first minutes following the trauma. Another limitation of the study was the heterogeneous nature of the patients involving a variety of cerebral injuries. In the present study, we did not report not any physical finding related to the complications caused by the increase in ICP due to CC. Another limitation was that we did not report other non-brain traumatic injuries which may have impacted the ONSD data.

We conclude that CC increases the ICP and ONSD regardless of the type of cerebral trauma. Therefore, unnecessary CC applications should be avoided and the CCs should be removed without delay just after the suspicion of cervical trauma is eliminated.

\section{ACKNOWLEDGEMENTS}

We would like to thank the hospital staff who contributed to our study and the participants who agreed to participate.

\section{CONFLICT OF INTEREST}

The authors have not declared any conflict of interest.

\section{ETHICS APPROVAL}

Approved by the local ethics committee (Date: November, 30,2017 , approval register number 167).

\section{FUNDING INFORMATION}

The autors declared that this study was conducted without any financial support.

\section{REFERENCES}

[1] American College of Surgeons (ACS) Committee on Trauma. Advanced trauma life support (ATLS®) Student manual. 9th Ed. American College of Surgeons. 2012:148-205.

[2] Sundstrøm T, Asbjørnsen H, Habiba S, Sunde GA, Wester K. Prehospital use of cervical collars in trauma patients: a critical review. J neurotraum. 2014;31:531-40.

[3] Sparke A, Voss S, Benger J. The measurement of tissue interface pressures and changes in jugular venous parameters associated with cervical immobilisation devices: a systematic review. Scand J Trauma Resus. 2013;21:81.

[4] Davies G, Deakin C, Wilson A. The effect of a rigid collar on intracranial pressure. Injury. 1996;27:647-9.

[5] Hunt K, Hallworth S, Smith M. The effects of rigid collar placement on intracranial and cerebral perfusion pressures. Anaesthesia. 2001;56:511-3

[6] Mobbs RJ, Stoodley MA, Fuller J. Effect of cervical hard collar on intracranial pressure after head injury. ANZ journal of surgery. 2002;72:389-91.

[7] Gokcen E, Caltekin I, Savrun A, Korkmaz H, Savrun ST, et al. Alterations in optic nerve sheath diameter according to cerebrovascular disease sub-groups. Am J Emerg Med. 2017;35:1607-11.

[8] Hansen HC, Lagreze W, Krueger O, Helmke K. Dependence of the optic nerve sheath diameter on acutely applied subarachnoidal pressure - an experimental ultrasound study. Acta ophthalmologica. 2011;89:e528-32.

[9] Hansen HC, Helmke K. Validation of the optic nerve sheath response to changing cerebrospinal fluid pressure: ultrasound findings during intrathecal infusion tests. J Neurosurg. 1997;87:34-40.

[10] Amini A, Kariman H, Dolatabadi AA, Hatamabadi HR, Derakhshanfar $\mathrm{H}$, et al. Use of the sonographic diameter of optic nerve sheath to estimate intracranial pressure. Am J Emerg Med. 2013;31:236-9.

[11] Sahoo SS, Agrawal D. Correlation of optic nerve sheath diameter with intracranial pressure monitoring in patients with severe traumatic brain injury. The Indian Journal of Neurotrauma. 2013;10:9-12.

[12] Hansen HC, Helmke K. The subarachnoid space surrounding the optic nerves. An ultrasound study of the optic nerve sheath. Surg Radiol Anat. 1996;18:323-8

[13] Qayyum H, Ramlakhan S. Can ocular ultrasound predict intracranial hypertension? A pilot diagnostic accuracy evaluation in a UK emergency department. Eur J Emerg Med. 2013;20:91-7.

[14] Frumin E, Schlang J, Wiechmann W, Hata S, Rosen S, et al. Prospective analysis of single operator sonographic optic nerve sheath diameter measurement for diagnosis of elevated intracranial pressure. Western J Emerg Med. 2014;15:217-20.

[15] Woster CM, Zwank MD, Pasquarella JR, Wewerka SS, Anderson JP, et al. Placement of a cervical collar increases the optic nerve sheath diameter in healthy adults. Am J Emerg Med. 2018;36:430-4.

[16] Ohle R, McIsaac SM, Woo MY, Perry JJ. Sonography of the Optic Nerve Sheath Diameter for Detection of Raised Intracranial Pressure Compared to Computed Tomography: A Systematic Review and Meta-analysis. J Ultras Med. 2015;34:1285-94.

[17] Kimberly HH, Shah S, Marill K, Noble V. Correlation of optic nerve sheath diameter with direct measurement of intracranial pressure. Acad Emerg Med. 2008;15:201-4. 
[18] Stone MB, Tubridy CM, Curran R. The effect of rigid cervical collars on internal jugular vein dimensions. Acad Emerg Med. 2010;17:1002.

[19] Karason S, Reynisson K, Sigvaldason K, Sigurdsson GH. Evaluation of clinical efficacy and safety of cervical trauma collars: differences in immobilization, effect on jugular venous pressure and patient comfort. Scand J Trauma Resus. 2014;22:37.

[20] Raphael J, Chotai R. Effects of the cervical collar on cerebrospinal fluid pressure. Anaesthesia. 1994;49:437-9.

[21] Kroeker J, Keith J, Carruthers H, Hanna C, Qureshi N, Calic M et al. Investigating the Time-lapsed Effects of Rigid Cervical Collars on the Dimensions of the Internal Jugular Vein. Clin Anat. 2019;32:196-200.

[22] Emmez ÖH, Egemen E. Kafa içi basınç artışı tedavisinde pratik yaklaşımlar. Yoğun Bakım Dergisi. 2010;9:77-84.

[23] Ho AM-H, Fung K, Joynt GM, Karmakar MK, Peng Z. Rigid cervical collar and intracranial pressure of patients with severe head injury. $\mathrm{J}$ Trauma Acute Care. 2002;53:1185-8.

How to cite this article: Tamer Colak, Kaan Celik. The Association between Cervical Collar and Intracranial Pressure Measured by the Optic Nerve Sheath Diameter in Trauma Patients Refered to the Emergency Department. Signa Vitae. 2020;16(1):89-95. doi:10.22514/sv.2020.16.0012. 